\title{
Exploring Twinning in the CSD
}

\author{
V Young ${ }^{1}$, B Foxman ${ }^{2}$ \\ ${ }^{1}$ Dept of Chemistry, Univ of Minnesota, Minneapolis, MN, ${ }^{2}$ Chemistry MS015, Brandeis Univ. \\ vyoung@umn.edu
}

The 2020 Version 5.41 (November 2019) of the Cambridge Structural Database1 now boasts 1,000,000-plus crystal structures of organic, organometallic, and other related materials containing organic molecular fragments. Of these, 19,156 are cited as being twins of some sort. Quite often the type of twin is omitted from the description altogether. We have embarked on a detailed study of this group of crystal structures to learn various hidden details of interest. First of all, while the descriptor twin is often listed, we often find the respective authors determined experimentally that specimen to be a single crystal (actual inversion twin by use of the Flack X parameter) within accepted standard uncertainties. On the other hand, the absence of modifiers; e.g. inversion, merohedral, non-merohedral, and recticular; reduces the overall value to the database and the additional research opportunities it could provide. The present study will focus on crystal structures within the triclinic crystal system where twin is indicated or where some sort of twin should be indicated. The vast majority of these are $Z^{\prime}>1$ crystal structures. Our study will also focus on two major subgroups, transformational- and growth twins, in order to learn more about their composition planes and putative, unexplored/unreported enantiotropic phase transitions. Several useful software tools will be presented in use with worked examples. 1. The Cambridge Structural Database, C. R. Groom, I. J. Bruno, M. P. Lightfoot and S. C. Ward, Acta Cryst. (2016). B72, 171-179 DOI: 10.1107/S2052520616003954

Acta Cryst. (2020). A76, a16 